\title{
Impaired Hand Dexterity Function in a Non-human Primate Model with Chronic Parkinson's Disease
}

\author{
Jincheol Seo ${ }^{1,2 \dagger}$, Jinyoung Won ${ }^{1 \dagger}$, Keonwoo Kim ${ }^{1,2 \dagger}$, Junghyung Park ${ }^{1}$, Hyeon-Gu Yeo ${ }^{1,3}$, \\ Yu Gyeong Kim ${ }^{1,3}$, Seung Ho Baek ${ }^{1}$, Hoonwon Lee ${ }^{4}$, Chang-Yeop Jeon ${ }^{1}$, Won Seok Choi ${ }^{1}$, Sangil Lee ${ }^{5}$, \\ Ki Jin Kim ${ }^{5}$, Sung-Hyun Park ${ }^{1}$, Yeonghoon Son ${ }^{5}$, Kang Jin Jeong ${ }^{1}$, Kyung Seob Lim ${ }^{6}$, Philyong Kang ${ }^{6}$, \\ Hwal-Yong Lee ${ }^{6}$, Hee-Chang Son ${ }^{6}$, Jae-Won Huh ${ }^{1,3}$, Young-Hyun Kim ${ }^{1,3}$, Dong-Seok Lee ${ }^{2}$, \\ Sang-Rae Lee ${ }^{1,3}$, Ji-Woong Choi ${ }^{7,8 *}$ and Youngjeon Lee ${ }^{1,3 *}$ \\ ${ }^{1}$ National Primate Research Center, Korea Research Institute of Bioscience and Biotechnology (KRIBB), Cheongju 28116, \\ ${ }^{2}$ School of Life Sciences, BK21 Plus KNU Creative BioResearch Group, Kyungpook National University, Daegu 41566, \\ ${ }^{3}$ Department of Functional Genomics, KRIBB School of Bioscience, Korea University of Science and Technology, Daejeon \\ $34113,{ }^{4}$ School of Biological Sciences, Seoul National University, Seoul 08826, ${ }^{5}$ Primate Resource Center, KRIBB, Jeongeup \\ 56216, ${ }^{6}$ Futuristic Animal Resource \& Research Center, KRIBB, Cheongju 28116, ${ }^{7}$ Brain Engineering Convergence Research \\ Center, Daegu Gyeongbuk Institute of Science \& Technology (DGIST), Daegu 42988, ${ }^{8}$ Department of Information and \\ Communication Engineering, DGIST, Daegu 42988, Korea
}

\begin{abstract}
Symptoms of Parkinson's disease (PD) caused by loss of dopaminergic neurons are accompanied by movement disorders, including tremors, rigidity, bradykinesia, and akinesia. Non-human primate (NHP) models with PD play an essential role in the analysis of PD pathophysiology and behavior symptoms. As impairments of hand dexterity function can affect activities of daily living in patients with PD, research on hand dexterity function in NHP models with chronic PD is essential. Traditional rating scales previously used in the evaluation of animal spontaneous behavior were insufficient due to factors related to subjectivity and passivity. Thus, experimentally designed applications for an appropriate apparatus are necessary. In this study, we aimed to longitudinally assess hand dexterity function using hand dexterity task (HDT) in NHP-PD models. To validate this assessment, we analyzed the alteration in Parkinsonian tremor signs and the functionality of presynaptic dopaminergic neuron using positron emission tomography imaging of dopamine transporters in these models. In addition, a significant inverse correlation between HDT and DAT level was identified, but no local bias was found. The correlation with intention tremor signs was lower than the resting tremor. In conclusion, the evaluation of HDT may reflect behavioral symptoms of NHP-PD models. Furthermore, HDT was effectively used to experimentally distinguish intention tremors from other tremors.
\end{abstract}

Key words: Parkinson's disease, Non-human primate, Hand dexterity function, Hand dexterity task, Tremor

\section{INTRODUCTION}

Submitted September 2, 2020, Revised September 28, 2020,

Accepted October 5, 2020

* To whom correspondence should be addressed.

Youngjeon Lee, TEL: 82-43-240-6316, FAX: 82-43-240-6309

e-mail:neurosci@kribb.re.kr

Ji-Woong Choi, TEL: 82-53-785-6311, FAX: 82-53-785-6309

e-mail:jwchoi@dgist.ac.kr

"These authors contributed equally to this work.
Symptoms of Parkinson's disease (PD) caused by loss of dopaminergic neurons are accompanied by movement disorders, including tremors, rigidity, bradykinesia, and akinesia [1,2]. As impairments of hand dexterity function can affect activities of daily living in patients with PD, such as buttoning a shirt or picking up a fork, recent studies have attempted to identify related pathological processes, such as apraxia, to develop a treatment strategy [3, 
4]. Various methods have been developed to assess hand dexterity dysfunction in patients with PD [5-7].

Non-human primate (NHP) of PD models play an essential role in the analysis of PD pathophysiology and behavior symptoms [8]. However, methods that can be applied to NHP models with PD are limited and confined to those based on visual phenotype observations. Traditional rating scales previously used in the evaluation of animal spontaneous behavior were problematic due to factors related to subjectivity and passivity [9]. Observation methods can neither eliminate idiosyncrasies of each observer's subjective judgment (subjectivity) nor separate bradykinesia from temporary spontaneous laziness of the subjects (passivity). In particular, distinguishing parkinsonian symptoms, such as bradykinesia, from spontaneous laziness in NHP-PD models was challenging [9].

To overcome these challenges, experimentally designed applications of an appropriate apparatus are necessary. An apparatus for the hand dexterity task (HDT) used in experimental NHP models, such as stroke, in recent studies [10-13], can analyze hand dexterity function to measure latency in retrieving food pellets from wells of various sizes and depths. The HDT method has been applied with the expectation that the latency to retrieve food will increase in proportion to the severity of PD symptoms, including bradykinesia, rigidity, and intention tremor in voluntary movements. A performance assessment based on appetite, contrary to passive observation, can evaluate symptoms that occur during voluntary movement and can resolve confounding behavior issues associated with spontaneous laziness.

Furthermore, we believe that an apparatus for the HDT could be used to distinguish intention tremors from other tremors. We suggested that the latency to retrieve food would increase in proportion to the intention tremor severity in voluntary movements. Although Parkinsonian tremors are key symptoms of the disease, understanding the development of tremors remains unclear. In addition, a research on the differentiation between resting and intention tremors in voluntary movements can be a clue for Parkinsonian tremors $[14,15]$.

In this study, we aimed to longitudinally assess hand dexterity function using the HDT in a previously developed 1-methyl4-phenyl-1,2,3,6-tetrahydropyridine (MPTP)-induced NHP model for chronic PD [9]. To validate this assessment, we analyzed the alteration of Parkinsonian behavior score, including signs of tremor and positron emission tomography (PET) imaging of dopamine transporters (DAT), with $\left[{ }^{18} \mathrm{~F}\right] \mathrm{N}$-(3-fluoropropyl)-2 $\beta$ carboxymethoxy-3 $\beta$-(4-iodophenyl) nortropane $\left({ }^{18} \mathrm{~F}\right.$-FP-CIT).

\section{MATERIALS AND METHODS}

\section{Experimental animals and MPTP administration}

Seven female adult cynomolgus monkeys (Macaca fascicularis) were employed in this study. Four monkeys were intramuscularly injected daily with MPTP $(0.2 \mathrm{mg} / \mathrm{kg}$; Sigma-Aldrich, St. Louis, MO, USA) dissolved in saline into the left femoral region while three monkeys were injected with saline. The design for MPTP injections was based on individual behavioral quantification to induce stable chronic PD models [9]. The total MPTP dose was commensurate with each individual animal's behavioral quantification. An overview of the design is presented in Fig. 1A, Table 1.

\section{Ethical statement}

This animal experiment was approved by the Korea Research Institute of Bioscience and Biotechnology Institutional Animal Care and Use Committee (Approval No. KRIBB-AEC-16068) [9, 1621]. MPTP treated NHP models are used to reproduce essential behavior PD signs, since they show a higher similarity to human patients with PD than other animals [22-25]. Based on previous studies, the individual response of cynomolgus to MPTP varies [9, 26]. Therefore, we determined that at least 7 MPTP cynomolgus monkeys ( 4 treatment, 3 saline controls) were needed to achieve the study purpose. To reduce unnecessary sacrifice, we used PET to identify damage to the cerebral dopaminergic system.

The monkeys originated from the Zhaoqing Laboratory Animal Research Centre (Guangdong Province, China) and were maintained in individual indoor cages at the National Primate Research Center at the Korea Research Institute of Bioscience and Biotechnology (KRIBB), as previously reported In brief, the experimental animals housed in cages faced each other in double columns. So, the animals had visual contact and voice interaction to neighbors without physical contact to avoid the damage to other individuals through the excretion of MPTP metabolites. The dimensions of cages are $60 \mathrm{~cm} \times 80 \mathrm{~cm} \times 80 \mathrm{~cm}$. They were fed commercial monkey chow (Teklad $2050^{\mathrm{TM}}$, Envigo, USA) supplemented by various fruits and were given water ad libitum. They were also supplied various rubber and plastic toys and fruits for enrichments. If monkeys do not have an interest in toys, new shape and size of toys were put in cages. Environmental conditions were maintained a temperature of $24 \pm 2$ degrees Celsius, a relative humidity of $50 \pm 5 \%$, and a $12 \mathrm{~h}$ light $/ 2 \mathrm{~h}$ dark cycle. The attending veterinarian monitored the monkeys' health in accordance with the recommendations of the Weatherall [27] report on the use of nonhuman primates in research. Animal health monitoring was performed by microbiological tests including B virus, simian retrovirus (SRV), simian immunodeficiency virus (SIV), simian virus 40 (SV40), 


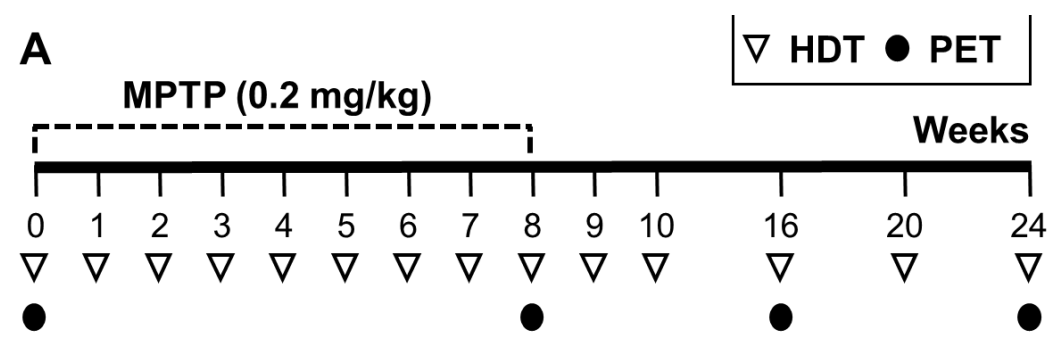

B

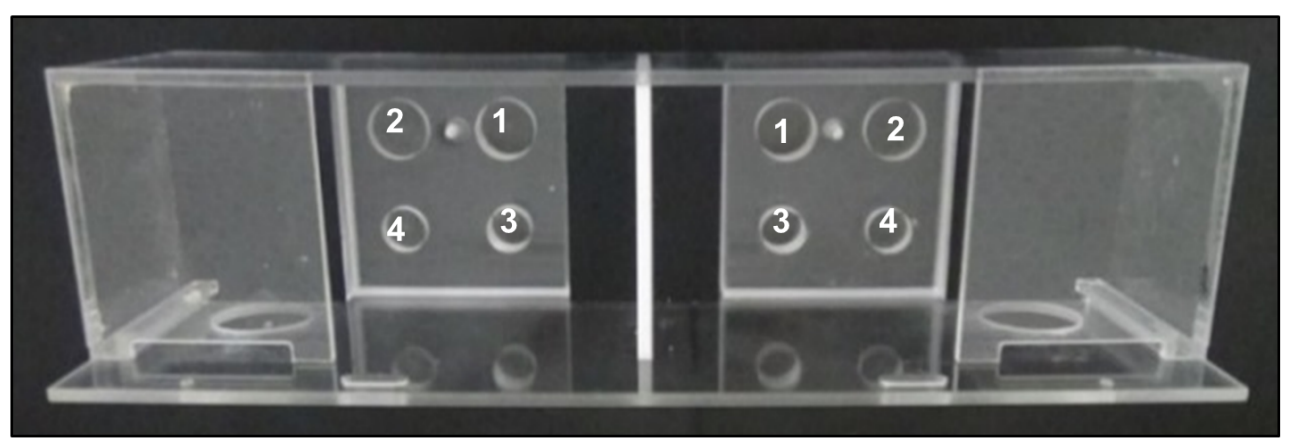

C

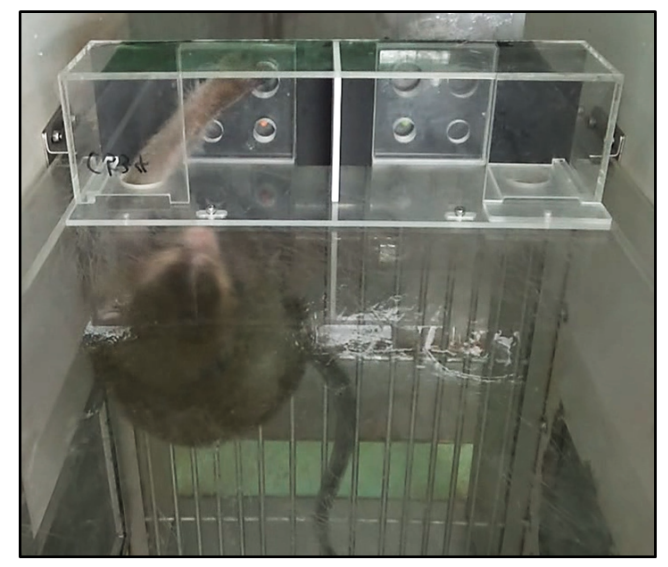

Right hand

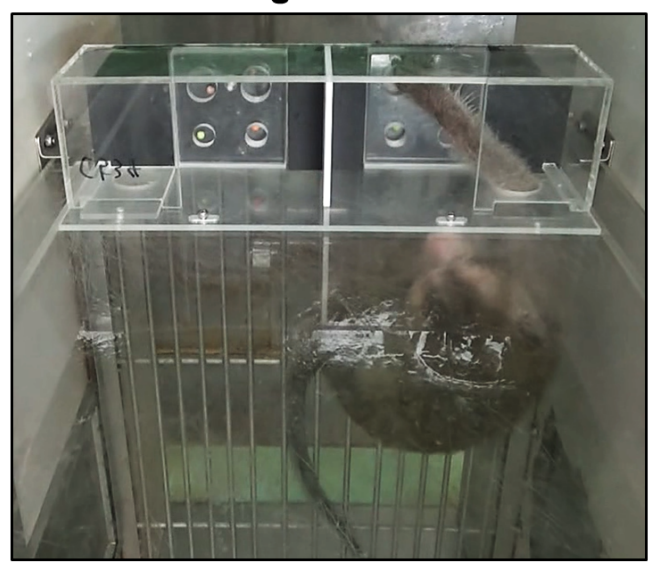

Fig. 1. Introduction of the hand dexterity task (HDT) for cynomolgus monkeys. (A) Experimental overview of MPTP administration, hand dexterity task (HDT) and positron emission tomography (PET). (B) Representative images showing an HDT apparatus. (C) Captured images during HDT performance for each hand. and simian T-cell lymphotropic virus (STLV) at once a year, as described previously [28].

\section{Hand dexterity task}

A standard HDT instrument was used for the evaluation of hand dexterity dysfunction caused by PD. All monkeys were trained to retrieve $190 \mathrm{mg}$ of pellets (Fruit Crunchies, Bio Serv, USA) from 4 wells of various depths and diameters located in a tray on the left or right side of the apparatus, as explained in detail in previous studies [10-13, 21, 29]. The 4 wells had the following characteristics: Well 1=wide and deep, Well 2=wide and shallow, Well 3 =narrow and deep, Well $4=$ narrow and shallow; diameter: narrow $=1.9 \mathrm{~cm}$, wide $=2.5 \mathrm{~cm}$; depth: shallow $=0.95 \mathrm{~cm}$, deep $=1.6$ $\mathrm{cm}$ (Fig. 1B, C). The different hole sizes required different levels of finger dexterity of the monkey to retrieve the food pellets. The testing was conducted before breakfast in order to increase each animal's appetite. The performance assessment based on appetite made it possible to exclude spontaneous laziness of the animals as a confounding factor. Eight trials were conducted ( 4 trials per hand) on the trial day once a week, and each trial was limited to 2 min. Three training sessions were conducted for 3 weeks before MTPT or saline injections. The same sequence was maintained for all HDTs. The results of the third weekly trial before MPTP treatment on both hands of each monkey (baseline) was used as a comparison criterion for the latency to retrieve pellets after MPTP administration on the HDT. An overall process sequence was recorded using a digital video camera (HDR-CX405, Sony Corp., Japan) fixed on the top of the cage. The recorded video was used 
Table 1. Overview of MPTP and saline injections

\begin{tabular}{|c|c|c|c|c|c|c|}
\hline Group & Label & Age (years) & Body weight (kg) & Dose (mg/kg) & Number of doses & Total dose (mg/kg) \\
\hline \multirow[t]{4}{*}{$\operatorname{MPTP}(\mathrm{n}=4)$} & $\mathrm{C} 1$ & 8 & 2.55 & 0.2 & 16 & 3.2 \\
\hline & $\mathrm{C} 2$ & 7 & 2.84 & & 14 & 2.8 \\
\hline & $\mathrm{C} 3$ & 8 & 2.80 & & 35 & 7.0 \\
\hline & $\mathrm{C} 4$ & 6 & 2.82 & & 17 & 3.4 \\
\hline \multirow[t]{3}{*}{ Control $(\mathrm{n}=3)$} & $\mathrm{C} 7$ & 7 & 2.85 & Saline $(0.6 \sim 0.7 \mathrm{ml})$ & 20 & \\
\hline & $\mathrm{C} 8$ & 4 & 3.29 & & 20 & \\
\hline & C9 & 6 & 2.88 & & 20 & \\
\hline
\end{tabular}

MPTP, 1-methyl-4-phenyl-1,2,3,6-tetrahydropyridine.

to measure pellet retrieval latency from each of the four wells. The latency time indicated the time to return from the passage hole with retrieved pellet. Latency cut-off in each well was $15 \mathrm{~s}$. In order to perform a correlation analysis on the HDT and DAT level at 8, 16 and 24 weeks after MPTP injections, the results of ${ }^{18}$ F-FP-CIT binding potential in PET scan of previous studies were used [9].

The apparatus was made of polycarbonate. The thickness of the material was $20 \mathrm{~mm}$ or $30 \mathrm{~mm}$. The device was safely secured by stainless steel holders and screws, and blocked to outside contact by a transparent PC front panel, except for the device passage. Only one observer performed the whole test process and video recordings. The observer placed the food reward in the holes, opened the passage cover, and immediately left the test room. In order to minimize observer interference, the latency time to retrieve the food was measured from the moment the monkey's hand entered the passage.

\section{Parkinsonian tremor assessment and global activity}

The intention tremor assessment was utilized to calculate signs of tremor using the recorded video during HDT performance using the tremor item of Kurlan scale [30,31]. This scale rates 10 parkinsonian motor symptoms: facial expression ( $0 \sim 3)$; resting tremor ( $0 \sim 3)$; action or intention tremor $(0 \sim 3)$; posture $(0 \sim 2)$; gait $(0 \sim 3)$; bradykinesia $(0 \sim 4)$; balance coordination $(0 \sim 3)$; gross motor skills of upper limb (0 3) and lower limb (0 3), and defense reaction $(0 \sim 2)$. The minimum score is 0 , which indicates a normal degree of movement, while the maximum total score is 29. Data regarding the spontaneous behavior in individual home cages was collected using a digital video camera (HDR-CX405, Sony Corp., Japan) in front of the home cage. A total of $240 \mathrm{~min}$ of behavior was recorded consecutively (12:00 4:00 p.m.) for each monkey after HDT. The resting tremor of the monkeys was evaluated according to a rating scale for monkey PD models through a $5 \mathrm{~min}$ video recording analyzed every $60 \mathrm{~min}$ for a total of $240 \mathrm{~min}$; the tremors were then scored based on the Kurlan scale, as in a previous study [9]. The evaluation was performed by three trained examiners who were not informed of the experimental design. In addition, the monkeys' behavior was evaluated using global activity (Smart 3.0, Panlab S.L., Barcelona, Spain) via analysis of video recordings conducted for a total duration of $240 \mathrm{~min}$. This software reflects quantitative changes in movements by counting the rates of change of all pixels between sequentially captured video images of the $240 \mathrm{~min}$ of recordings for each experimental animal $[9,20]$.

\section{Positron emission tomography (PET) imaging protocol}

All PET images were acquired using a PET/computed tomography (CT) scanner (nanoScan PET-CT, Mediso Ltd., Budapest, Hungary). $\left[{ }^{18} \mathrm{~F}\right] \mathrm{N}$-(3-fluoropropyl)-2 $\beta$-carboxymethoxy-3 $\beta$ (4-iodophenyl) nortropane $\left({ }^{18} \mathrm{~F}\right.$-FP-CIT) PET imaging was performed to evaluate the expression of dopamine transporters [32]. ${ }^{18}$ F-FP-CIT was obtained from DuChemBio Co., Ltd., Daejeon, Korea. We intravenously injected $185 \mathrm{MBq}{ }^{18}$ F-FP-CIT in $1.5 \mathrm{~mL}$ saline via the saphenous vein after a CT was conducted for attenuation correction. Concurrently, PET acquisition with a $400 \times 400$ matrix size in list mode was performed for $120 \mathrm{~min}$ following a 39-frame dynamic sequence: $15 \mathrm{~s} \times 8,30 s \times 6,60 s \times 5,120 s \times 5$, $300 s \times 10$, and $600 s \times 5$. Images were reconstructed using a threedimensional ordered subsets expectation maximization algorithm (Tera-Tomo, Mediso Ltd., Hungary) and post-processed using syngo VG51C software (Siemens Medical Solutions, Erlangen, Germany) to visualize multiplanar fusion images. The reconstructed voxel values in each frame were reported in standard uptake value from body weight and corrected for radioactive decay to the time of injection; voxel dimensions were $0.4 \times 0.4 \times 0.4 \mathrm{~mm}^{3}$. The final three frames corresponding to the $90 \sim 120$ min following injection were used for dynamic analysis as the specific striatal uptake reached the maximum value from baseline.

\section{Statistical analysis}

All statistical analyses were performed using the Statistical Package for the Social Sciences for Windows, Version, 18.0 (SPSS Inc., Chicago, IL). Results of the HDT and PET imaging were analyzed by comparing to baseline, using repeated measures analysis of 

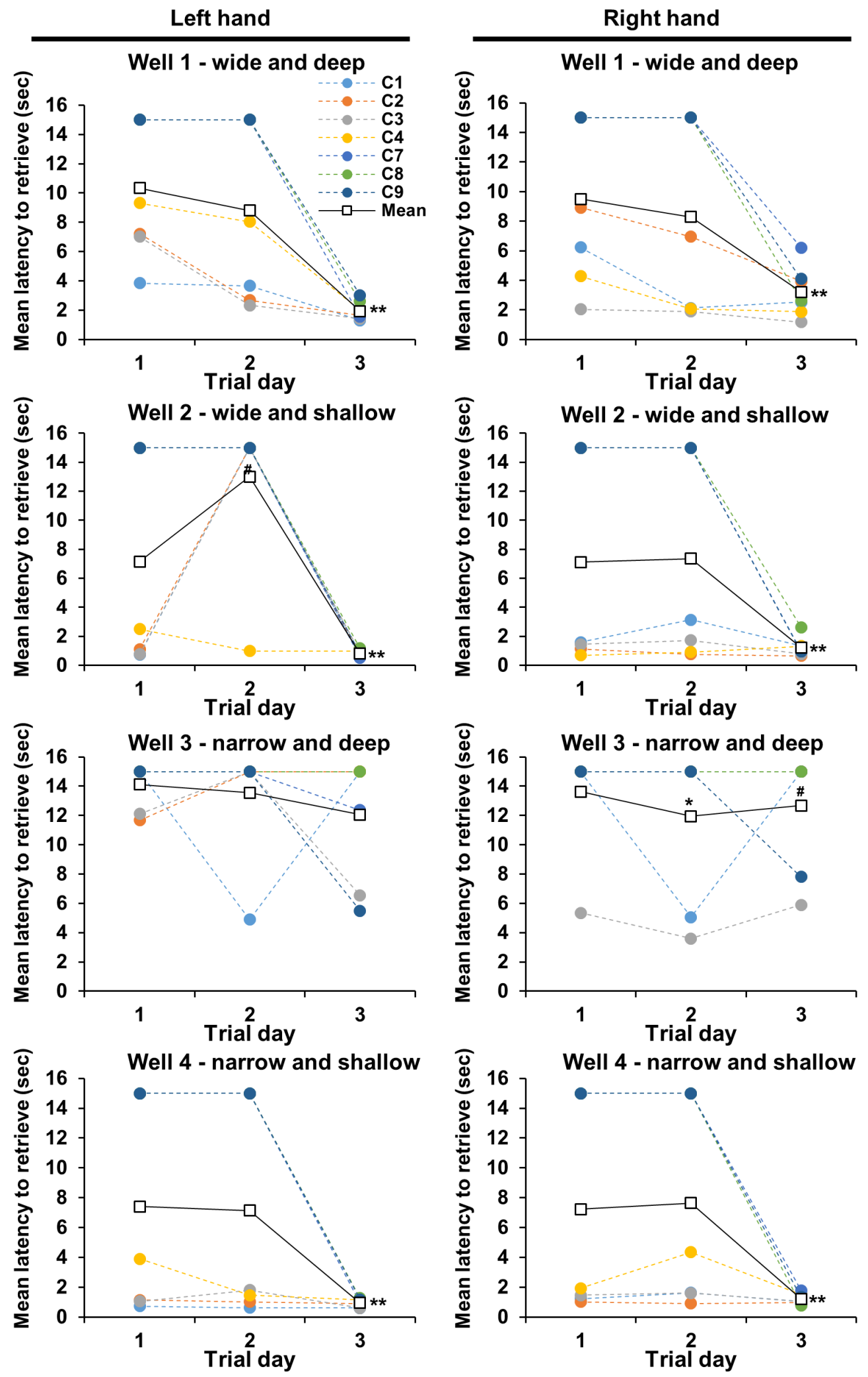

Fig. 2. The cynomolgus monkeys adapted to HDT for 3 trial days. All cynomolgus monkeys had adapted to Well 1,2, and 4 of the HDT for 3 trial days $(n=7)$. Empty square box symbol represents average value. ${ }^{*} \mathrm{p}<0.05$, ${ }^{*} \mathrm{p}<0.01$ vs. 1 trial day, ${ }^{* *} \mathrm{p}<0.01$ vs. 1 and 2 trial day respectively; repeated measures ANOVA. 
variance ANOVA. Tremor scores were analyzed by comparing to baseline using the Friedman non-parametric repeated measures test. Linear regressions were used to analyze the relationship between HDT, PET imaging results, and tremor scores. $\mathrm{p}<0.05$ was considered statistically significant.

\section{RESULTS}

\section{Cynomolgus monkeys adapted to HDT for 3 trial days}

Three training sessions were conducted for 3 weeks to train the
HDT before MTPT or saline injections. All cynomolgus monkeys had significantly adapted to Well 1,2, and 4 of HDT for 3 trial days. The latency of each wells on three trial days of baseline test recorded was as follows, mean ( \pm standard deviation, $p$ values vs. 1 and 2 trial day respectively): Well 1 left $=1.94( \pm 0.65, \mathrm{p}=0.001,0.001)$ sec, Well 1 right $=3.21( \pm 1.68, \mathrm{p}=0.001,0.001) \mathrm{sec}$, Well 2 left $=0.80$ $( \pm 0.22, \mathrm{p}=0.001,0.001) \mathrm{sec}$, Well 2 right $=1.23( \pm 0.66, \mathrm{p}=0.001$, $0.001) \mathrm{sec}$, Well 3 left $=12.06( \pm 4.25, \mathrm{p}=0.015,0.068) \mathrm{sec}$, Well 3 right $=12.67( \pm 4.02, \mathrm{p}=0.064,0.343) \mathrm{sec}$, Well 4 left $=0.95( \pm 0.26$, $\mathrm{p}=0.001,0.001) \mathrm{sec}$, Well 4 right $=1.21( \pm 0.35, \mathrm{p}=0.001,0.001) \mathrm{sec}$

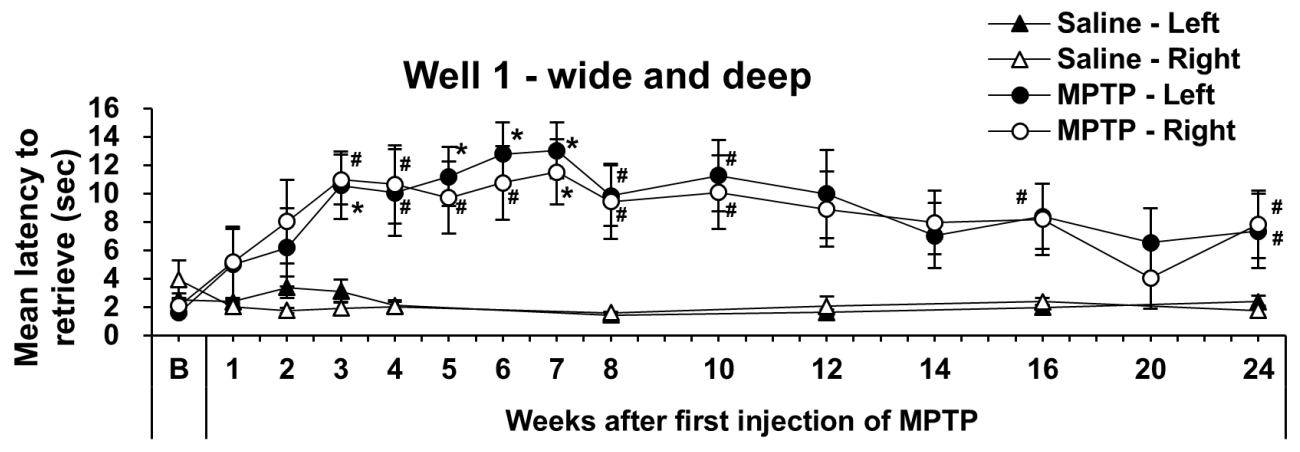

Well 2 - wide and shallow

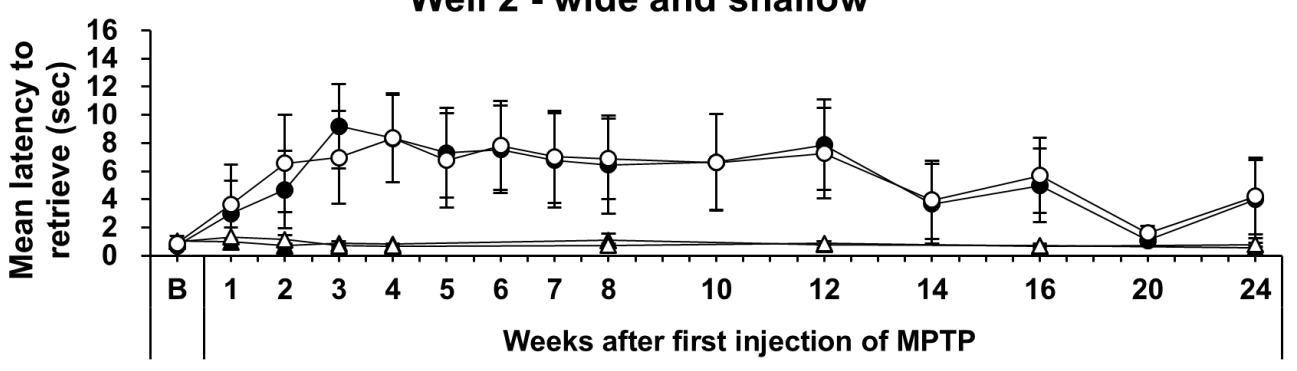

Well 3 - narrow and deep

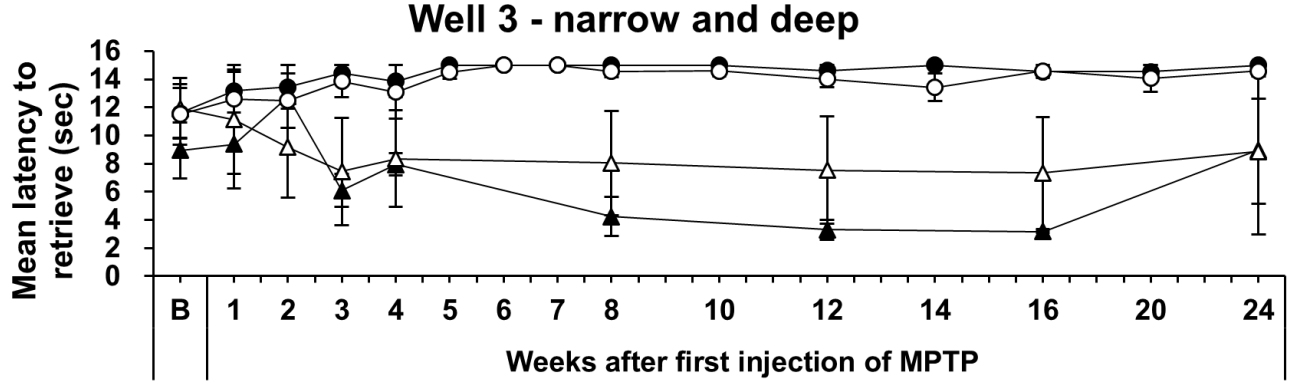

Well 4 - narrow and shallow

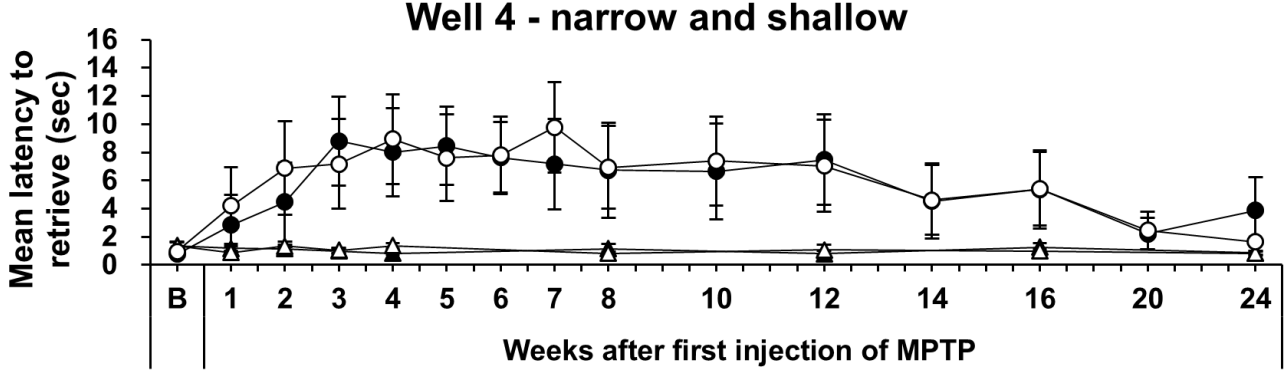

Fig. 3. Mean latency to retrieve pellets on the hand dexterity task (HDT). HDT was used to compare movements from week 1 after the first MPTP administration; latency to retrieve pellets from Well 1 in the HDT task was significantly increased for both hands, compared with baseline, and remained at that level until 24 weeks after the first administration. ${ }^{*} \mathrm{p}<0.05,{ }^{*} \mathrm{p}<0.01$ vs. baseline; repeated measures ANOVA. $\mathrm{B}$, baseline. 

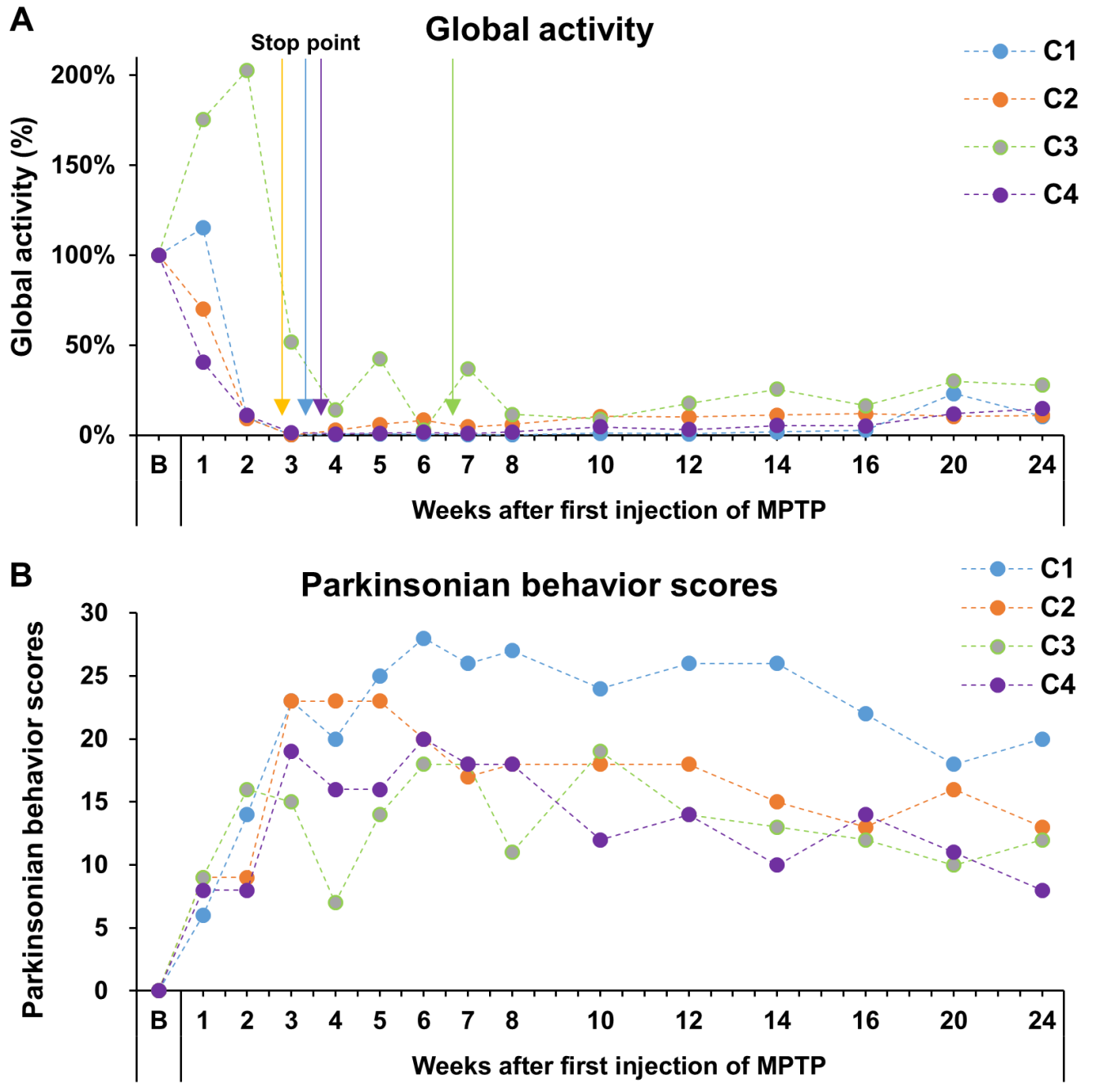

Fig. 4. Four cynomolgus showed PD behavioral signs after MPTP administration. (A) Global activity was reduced after the first MPTP administration. Each cynomolgus response to MPTP differed over time. The total MPTP dose was commensurate to each individual animal's global activity level. Arrow lines indicate the final timepoint of MPTP injection. (B) Histogram representing Parkinsonian behavior scores shows continuous parkinsonism. $(\mathrm{n}=4$, MPTP injected group; $\mathrm{B}$, baseline).

(Fig. 2). There was no significant difference in the performance between the left and right hands.

\section{Latency identified in the HDT significantly increased after MPTP injections}

To confirm functional hand dexterity impairment, we observed the latency of HDT before and after MPTP administration (Fig. 3). HDT was used to compare movements from week 1 after the first MPTP administration; latency to retrieve pellets from Well 1 in the HDT task was significantly increased for both hands, compared with baseline, and remained at that level until 24 weeks after the first administration (Fig. 3). The tendency of increased latency was also observed in Well 2 and 4; however, latency to retrieve pellets from other wells, except Well 1 of the HDT task, did not significantly change. According to global activity and Parkinsonian behavior scores, 4 cynomolgus showed behavioral PD signs after MPTP administration (Fig. 4).

\section{Tremor signs during HDT performance}

Neurological examinations of the signs of tremor were conducted using tremor scores based on the Kurlan scale [9, 30, 31]. Tremor assessments were evaluated by analyzing video recordings during HDT performance (Supplementary Video. 1 and 2) and spontaneous behavior in individual cages. The tremor score during spontaneous behavior in individual cages showed continuous signs of tremor from 3 weeks after the first MPTP administration (Fig. 5A). Tremor score recordings during HDT performance showed a tendency to increase, but it did not reach significance. There was a positive correlation between HDT and both tremor symptom levels (Fig. 5, R=0.33 0.64, p<0.01). Unexpectedly, HDT showed a higher correlation with tremor symptoms on spontaneous behavior compared to tremor symptoms on HDT performance. 

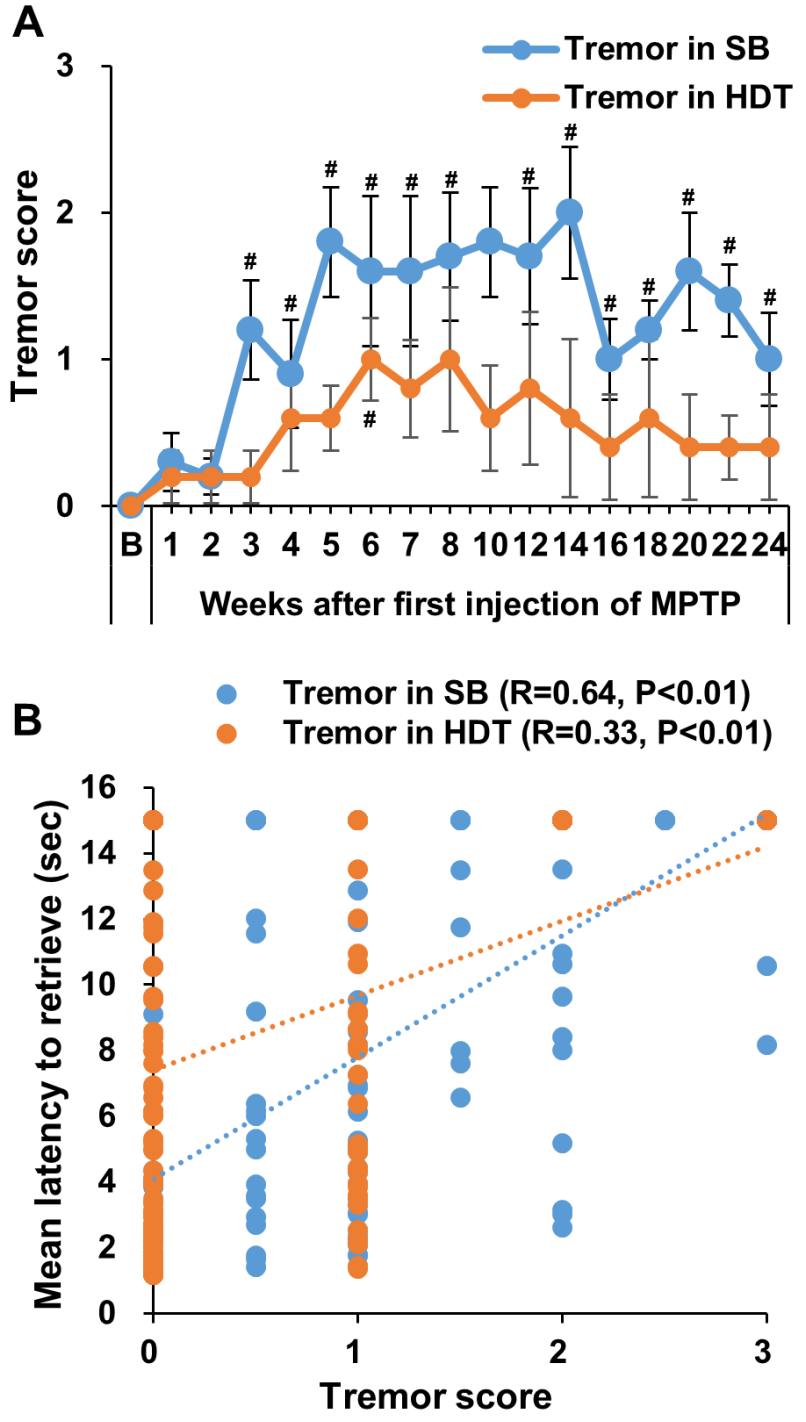

Fig. 5. Correlation between hand dexterity task (HDT) and signs of tremor. (A) Changes of tremors in spontaneous behavior (SB) and hand dexterity task (HDT) after MPTP administration. Tremors scores in SB remained high. (B) Positive correlations between average latencies of the left and right hand from Well 1 and the tremor score in $\mathrm{SB}(\mathrm{R}=0.64$, $\mathrm{p}<0.01)$ and HDT $(\mathrm{R}=0.33, \mathrm{p}<0.01)$. Trend lines: solid line, tremor in SB; dotted line, tremor in HDT. ${ }^{*} \mathrm{p}<0.05,{ }^{*} \mathrm{p}<0.01$ vs. baseline; repeated measures ANOVA. SB, spontaneous behavior; HDT, hand dexterity task; B, baseline.

\section{Significant inverse correlation between HDT and DAT lev- els but no local bias}

To analyze the correlation between HDT and DAT levels, linear regressions were performed using PET results from a previous study [9]. No local bias in the correlation between HDT and DAT levels the striatum was distinctively observed. Additionally, there was no difference between both hemispheres in DAT level (Fig. 6). However, a significant inverse correlation was found between the latency to complete HDT on Well 1 and the ${ }^{18} \mathrm{~F}$-FP-CIT BP in all striatum sub-regions on the opposite side (Fig. 7 and Table 2); monkeys with fewer striatal DAT tended to show poorer performance, indicating that HDT data reflects the level of striatal DAT.

\section{DISCUSSION}

The major finding of this study was that Well 1 in the HDT task was sufficiently sensitive to detect impairments of hand dexterity function induced by injection of MPTP. This study suggested that the HDT can investigate the behavior symptoms of NHPPD models. In addition, HDT can provide verifiable means to investigate parkinsonian hand dexterity function in NHP-PD models. As explained in previous studies [10], the different sized holes required the monkey to retrieve the food reward using different fingers and movements. The most efficient performance is attained utilizing precise control of the digits in a finger-thumb pincer fashion for each hole. However, this study did not show that the sensitivity of Well 1 , in comparison with other wells, was predominantly determined by the hole size or position. The tendency of increased latency was also observed in Well 2 and 4. However, there were not significantly increased. We considered that is not an appropriate difficulty level for NHP PD models. It was also considered that NHPs did not acquire sufficient proficiency in Well 3 compared to other wells.

As individual differences in proficiency and learning ability may affect the outcomes using this apparatus, a comparison with the baseline of the same animal is essential along with the control group of saline-injected animals. A minimum of three training sessions are required for accurate evaluation before the injection of MPTP. After three training sessions, all subjects showed constant latency to retrieve food (Fig. 2).

Furthermore, based on voluntary movements, we confirmed that this assessment resolved confounding issues between bradykinesia and spontaneous laziness. All monkeys injected with MPTP performed the HDT despite parkinsonian symptoms, such as bradykinesia and rigidity. The performance assessments based on appetite and foraging habits made it possible to exclude spontaneous laziness in the experimental animals. This method, therefore, is helpful not only to overcome the passivity of traditional observation evaluations but also aid in the investigation of hand dexterity functions in voluntary movements (Fig. 3).

In addition, we were able to distinguish intention tremor from other tremors during HDT performance (Supplementary Video. 1 and 2). Although controversial, movements that occur during appetite-based HDT tasks can be considered goal-directed movements. Hence, we considered that signs of tremor during the 
A Weeks after first MPTP administration

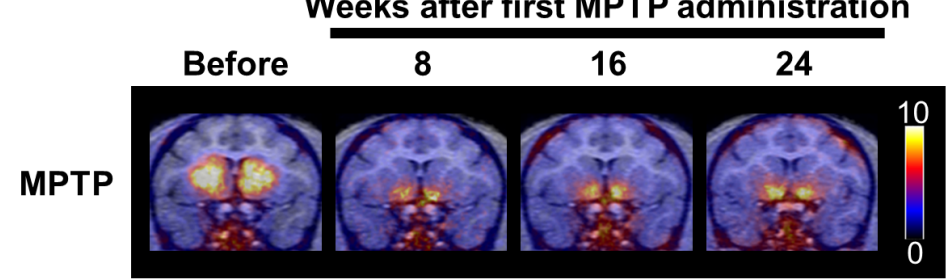

B 8 Before $\$ 8 \quad \square 16 \quad \square 24$ weeks after first MPTP administration

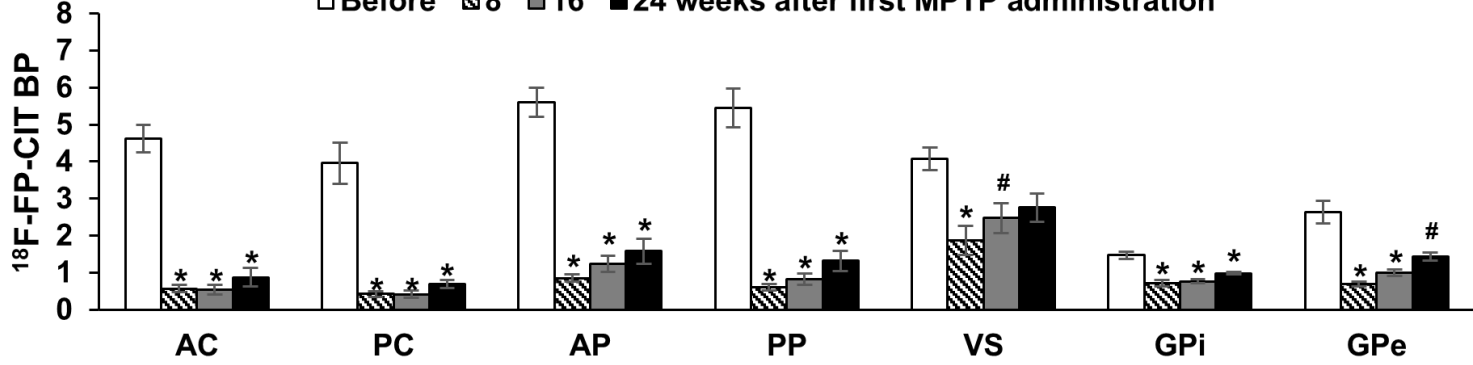

C
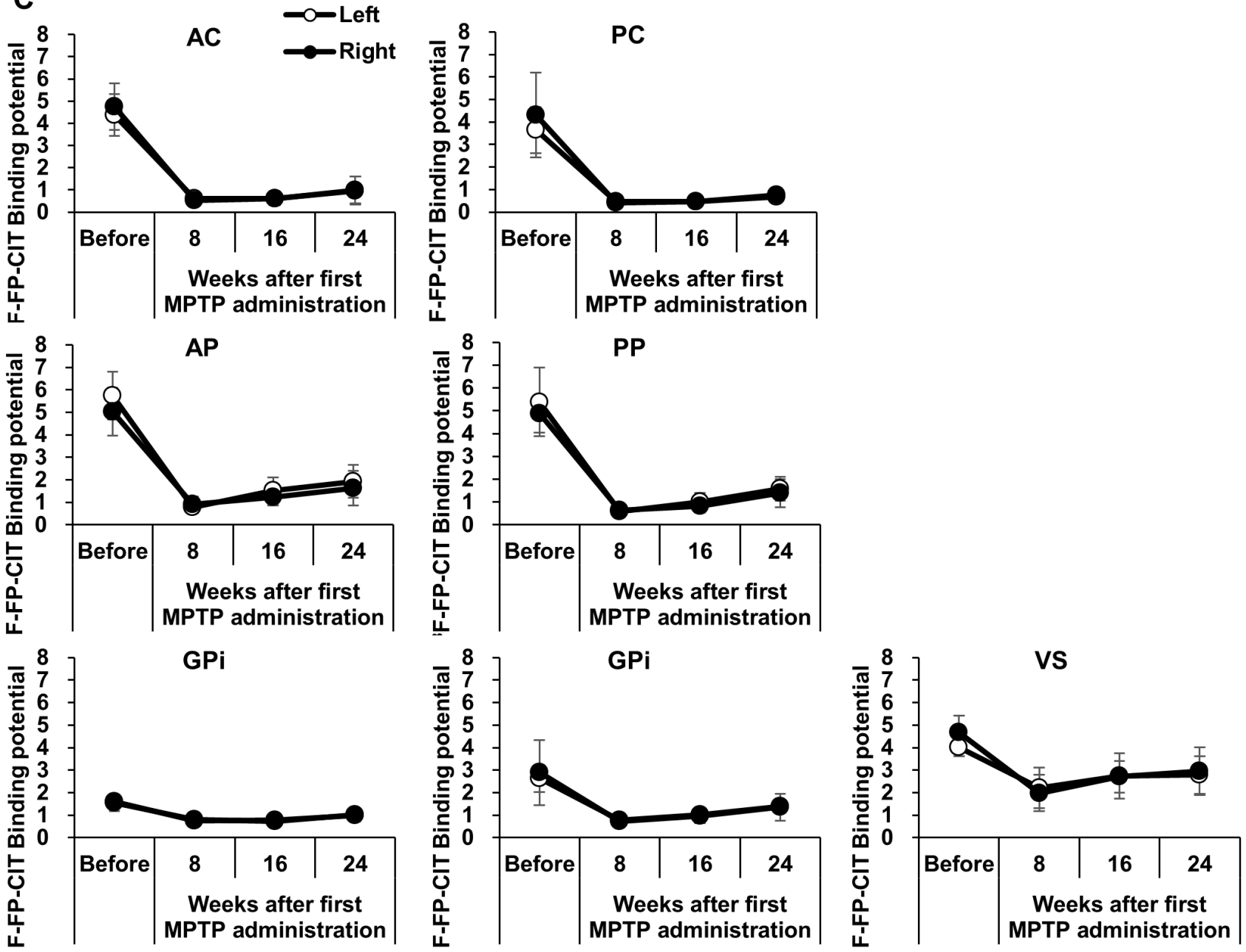

Fig. 6. Correlation between hand dexterity task (HDT) and dopamine transporter (DAT) activity. (A) Representative ${ }^{18}$ F-FP-CIT positron emission tomography (PET) images to indicate DAT at 8, 16 and 24 weeks timepoints after MPTP injections. (B) Histogram representing ${ }^{18}$ F-FP-CIT binding potential (BP) in the MPTP-injection group. (C) Quantitative analysis using the ${ }^{18} \mathrm{~F}$-FP-CIT binding potential of positron emission tomography imaging showing presynaptic dopamine transporter level of left and right hemispheres separately in MPTP injected monkeys. AC, anterior caudate; PC, posterior caudate; AP, anterior putamen; PP, posterior putamen; VS, ventral striatum; GPi, globus pallidus interna; GPe, globus pallidus external. The clear bar indicates the level before MPTP injections in the MPTP injected group. 


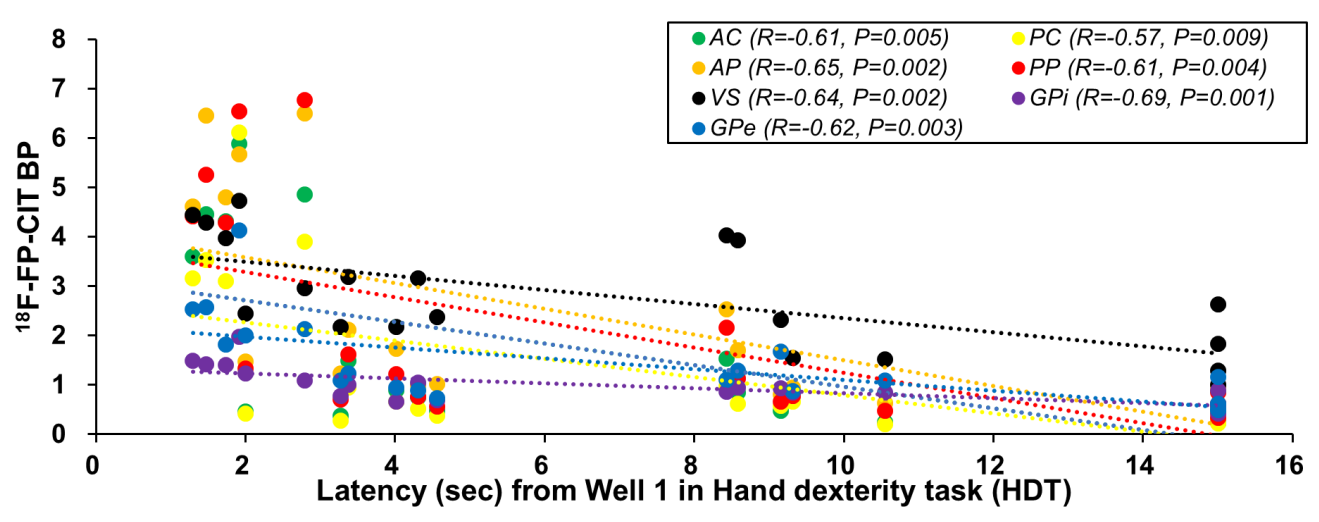

Fig. 7. Correlations between ${ }^{18} \mathrm{~F}$ FP-CIT (BP) levels with latency from Well 1 of the hand dexterity task in the MPTP-injection group at all matched PET timepoints. AC, anterior caudate; $\mathrm{PC}$, posterior caudate; $\mathrm{AP}$, anterior putamen; PP, posterior putamen; VS, ventral striatum; GPi, globus pallidus interna; GPe, globus pallidus external.

Table 2. Correlation coefficient (R) and p-value between ${ }^{18} \mathrm{~F}$-FP-CIT (BP) levels with each latency from all wells of the hand dexterity task in the MPTPinjection group at all matched PET timepoints

\begin{tabular}{|c|c|c|c|c|c|c|c|c|}
\hline & \multicolumn{2}{|c|}{ Well 1} & \multicolumn{2}{|c|}{ Well 2} & \multicolumn{2}{|c|}{ Well 3} & \multicolumn{2}{|c|}{ Well 4} \\
\hline & $\mathbf{R}$ & p & $\mathbf{R}$ & $\mathbf{p}$ & $\mathbf{R}$ & $\mathbf{p}$ & $\mathbf{R}$ & p \\
\hline $\mathrm{AC}$ & -0.61 & 0.005 & -0.34 & 0.082 & -0.17 & 0.250 & -0.37 & 0.067 \\
\hline PC & -0.57 & 0.009 & -0.31 & 0.106 & -0.13 & 0.300 & -0.33 & 0.089 \\
\hline $\mathrm{AP}$ & -0.65 & 0.002 & -0.36 & 0.073 & -0.24 & 0.171 & -0.38 & 0.062 \\
\hline PP & -0.61 & 0.004 & -0.33 & 0.090 & -0.22 & 0.194 & -0.35 & 0.076 \\
\hline VS & -0.64 & 0.002 & -0.25 & 0.161 & 0.13 & 0.306 & -0.27 & 0.136 \\
\hline GPi & -0.69 & 0.001 & -0.38 & 0.059 & -0.03 & 0.450 & -0.41 & 0.047 \\
\hline GPe & -0.62 & 0.003 & -0.37 & 0.063 & -0.11 & 0.328 & -0.39 & 0.057 \\
\hline
\end{tabular}

R, correlation coefficient; $\mathrm{p}$-value, significance probability; AC, anterior caudate; PC, posterior caudate; AP, anterior putamen; PP, posterior putamen; VS, ventral striatum; GPi, globus pallidus interna; GPe, globus pallidus external.

HDT tasks indicate intention tremor. Several clinical studies in patients with PD explained that resting and postural tremors during involuntary movements are more prominent than intention tremor during goal-directed movements [33-36]. However, studies pertaining to tremor signs mechanism remain insufficient, due to difficulty in animal model analysis $[37,38]$. Although several quantitative studies of tremor symptom in NHPs PD model were performed [39-42], it is challenging to distinguish rest tremor and other tremors for these animal models experimentally [38]. We believe that an attempt to distinguish type of tremor signs using HDT task for NHP models provides an important conceptual advance in the study of NHP-PD models and tremor signs.

In recent study of the marmoset MPTP model, the evidence of fine motor skills impairments and postural head tremor symptom were provided after MPTP injection using the object retrieval task with barrier detour (ORTBD) [43]. The ORTBD is similar to the HDT, but with a difference. Both tasks are the reward based behavioral testing system, but the HDT is more intuitive compared to ORTBD bypassing the barrier. The cognitive and problem solving skills are required to solve the detour part in the ORTBD, as evidenced in the studies [43-45]. Whereas, the HDT is able to evaluate the impairments of hand dexterity function independently.
We anticipated that it would be helpful to analyze impaired hand dexterity function in correlating analysis between the regional dopaminergic neuronal activity in the basal ganglia and tremor symptom of PD. With this method it was also expected that the latency to retrieve food would increase in proportion to the severity of intention tremor in voluntary movements. However, correlation with intention tremor signs was lower than the resting tremor in MPTP induced NHP-PD models (Fig. 5). The reason for this phenomenon is that the MPTP-induced PD models with impaired dopamine systems may indicate low relevance to intention tremor signs $[37,46]$. Furthermore, no local bias in the correlation between HDT and DAT levels in the region of striatum was distinctively observed, although this study showed that HDT latency weakly indicates levels of the DAT levels induced by MPTP (Fig. 6). According to previous studies, Parkinsonian tremor signs controlled by altered cerebral activity in the globus pallidus and the cerebello-thalamo-cortical circuits, distinct from the nigrostriatal pathway, could result in impaired hand dexterity function [47, 48]. In addition, a recent study described how a combination of both the basal ganglia and cerebellum plays a crucial role in tremor phenotypes [38]. The serotonergic system and gamma-aminobutyric acid (GABA)-ergic systems have also been implicated in PD 
[49-51]. Further precise comparisons of the behavior symptoms and neuropathology between NHP models may enhance better understanding of the mechanism of rest tremor in PD.

In conclusion, this study reinforces the fundamentals of mechanistic research for hand dysfunction in PD by applying HDT to NHP PD models with stable parkinsonian symptoms, thereby contributing to increase the quality of life for patients with PD. HDT can be helpful in the investigation of behavior symptoms of NHP-PD models. In addition, HDT enables experimental distinction between intention tremors and other tremors, and the correlation of HDT with intention tremor signs was lower than the resting tremor in NHP-PD model induced by MPTP.

\section{ACKNOWLEDGEMENTS}

This research was supported by the Bio \& Medical Technology Development Program of the National Research Foundation of Korea (NRF), funded by the Korean government, 2017M3A9G8084464 (Y.L.), 2017 M3C1B2085304 (Y.L.), and the Korea Research Institute of Bioscience and Biotechnology Research Initiative Program, KGC1022012 (Y.L.), KGM4622013 (J-W.H.), KGM5282022 (Y-H.K.). This work was performed at National Primate Research Center, Korea Research Institute of Bioscience and Biotechnology (KRIBB). The funders had no role in study design, data collection and analysis, decision to publish, or preparation of the manuscript. The funders had no role in study design, data collection and analysis, decision to publish, or preparation of the manuscript.

\section{SUPPLEMENTARY MATERIALS}

Supplementary Video. 1. The recorded video during HDT performance for each hand before MPTP injections.

Supplementary Video. 2. The recorded video during HDT performance for each hand at 8 weeks after MPTP injections.

\section{REFERENCES}

1. Kalia LV, Lang AE (2015) Parkinson's disease. Lancet 386:896912.

2. Poewe W, Seppi K, Tanner CM, Halliday GM, Brundin P, Volkmann J, Schrag AE, Lang AE (2017) Parkinson disease. Nat Rev Dis Primers 3:17013.

3. Vanbellingen T, Kersten B, Bellion M, Temperli P, Baronti F, Müri R, Bohlhalter S (2011) Impaired finger dexterity in Parkinson's disease is associated with praxis function. Brain Cogn 77:48-52.
4. Foki T, Pirker W, Klinger N, Geissler A, Rath J, Steinkellner T, Hoellinger I, Gruber S, Haubenberger D, Lehrner J, Pusswald G, Trattnig S, Auff E, Beisteiner R (2010) FMRI correlates of apraxia in Parkinson's disease patients OFF medication. Exp Neurol 225:416-422.

5. Wenzelburger R, Raethjen J, Löffler K, Stolze H, Illert M, Deuschl G (2000) Kinetic tremor in a reach-to-grasp movement in Parkinson's disease. Mov Disord 15:1084-1094.

6. Dahdal P, Meyer A, Chaturvedi M, Nowak K, Roesch AD, Fuhr P, Gschwandtner U (2016) Fine motor function skills in patients with Parkinson disease with and without mild cognitive impairment. Dement Geriatr Cogn Disord 42:127-134.

7. Pradhan SD, Brewer BR, Carvell GE, Sparto PJ, Delitto A, Matsuoka Y (2010) Assessment of fine motor control in individuals with Parkinson's disease using force tracking with a secondary cognitive task. J Neurol Phys Ther 34:32-40.

8. Emborg ME (2007) Nonhuman primate models of Parkinson's disease. ILAR J 48:339-355.

9. Seo J, Lee Y, Kim BS, Park J, Yang S, Yoon HJ, Yoo J, Park HS, Hong JJ, Koo BS, Baek SH, Jeon CY, Huh JW, Kim YH, Park SJ, Won J, Ahn YJ, Kim K, Jeong KJ, Kang P, Lee DS, Lim SM, Jin YB, Lee SR (2019) A non-human primate model for stable chronic Parkinson's disease induced by MPTP administration based on individual behavioral quantification. J Neurosci Methods 311:277-287.

10. Moore TL, Killiany RJ, Pessina MA, Moss MB, Rosene DL (2010) Assessment of motor function of the hand in aged rhesus monkeys. Somatosens Mot Res 27:121-130.

11. Moore TL, Killiany RJ, Pessina MA, Moss MB, Finklestein SP, Rosene DL (2012) Recovery from ischemia in the middleaged brain: a nonhuman primate model. Neurobiol Aging 33:619.e9-619.e24.

12. Moore TL, Pessina MA, Finklestein SP, Kramer BC, Killiany RJ, Rosene DL (2013) Recovery of fine motor performance after ischemic damage to motor cortex is facilitated by cell therapy in the rhesus monkey. Somatosens Mot Res 30:185196.

13. Moore TL, Pessina MA, Finklestein SP, Killiany RJ, Bowley B, Benowitz L, Rosene DL (2016) Inosine enhances recovery of grasp following cortical injury to the primary motor cortex of the rhesus monkey. Restor Neurol Neurosci 34:827-848.

14. Helmich RC, Hallett M, Deuschl G, Toni I, Bloem BR (2012) Cerebral causes and consequences of Parkinsonian resting tremor: a tale of two circuits? Brain 135(Pt 11):3206-3226.

15. Loane C, Wu K, Bain P, Brooks DJ, Piccini P, Politis M (2013) Serotonergic loss in motor circuitries correlates with severity of action-postural tremor in PD. Neurology 80:1850-1855. 
16. Kilkenny C, Browne WJ, Cuthill IC, Emerson M, Altman DG (2010) Improving bioscience research reporting: the ARRIVE guidelines for reporting animal research. PLoS Biol 8:e1000412.

17. Yeo HG, Lee Y, Jeon CY, Jeong KJ, Jin YB, Kang P, Kim SU, Kim JS, Huh JW, Kim YH, Sim BW, Song BS, Park YH, Hong Y, Lee SR, Chang KT (2015) Characterization of cerebral damage in a monkey model of Alzheimer's disease induced by intracerebroventricular injection of streptozotocin. J Alzheimers Dis 46:989-1005.

18. Lee Y, Kim YH, Park SJ, Huh JW, Kim SH, Kim SU, Kim JS, Jeong KJ, Lee KM, Hong Y, Lee SR, Chang KT (2014) Insulin/ IGF signaling-related gene expression in the brain of a sporadic Alzheimer's disease monkey model induced by intracerebroventricular injection of streptozotocin. J Alzheimers Dis 38:251-267.

19. Park J, Seo J, Won J, Yeo HG, Ahn YJ, Kim K, Jin YB, Koo BS, Lim KS, Jeong KJ, Kang P, Lee HY, Baek SH, Jeon CY, Hong JJ, Huh JW, Kim YH, Park SJ, Kim SU, Lee DS, Lee SR, Lee Y (2019) Abnormal mitochondria in a non-human primate model of MPTP-induced Parkinson's disease: Drp1 and CDK5/p25 signaling. Exp Neurobiol 28:414-424.

20. Seo J, Park J, Kim K, Won J, Yeo HG, Jin YB, Koo BS, Lim KS, Jeong KJ, Kang P, Lee HY, Choi WS, Baek SH, Jeon CY, Hong JJ, Huh JW, Kim YH, Park SJ, Kim SU, Lee DS, Lee SR, Lee Y (2020) Chronic infiltration of T lymphocytes into the brain in a non-human primate model of Parkinson's disease. Neuroscience 431:73-85.

21. Kim K, Jeon HA, Seo J, Park J, Won J, Yeo HG, Jeon CY, Huh JW, Kim YH, Hong Y, Choi JW, Lee Y (2020) Evaluation of cognitive function in adult rhesus monkeys using the finger maze test. Appl Anim Behav Sci 224:104945.

22. Blandini F, Armentero MT (2012) Animal models of Parkinson's disease. FEBS J 279:1156-1166.

23. Przedborski S, Jackson-Lewis V, Naini AB, Jakowec M, Petzinger G, Miller R, Akram M (2001) The Parkinsonian toxin 1-methyl-4-phenyl-1,2,3,6-tetrahydropyridine (MPTP): a technical review of its utility and safety. J Neurochem 76:1265-1274.

24. Porras G, Li Q, Bezard E (2012) Modeling Parkinson's disease in primates: the MPTP model. Cold Spring Harb Perspect Med 2:a009308.

25. Morin N, Jourdain VA, Di Paolo T (2014) Modeling dyskinesia in animal models of Parkinson disease. Exp Neurol 256:105-116.

26. Potts LF, Wu H, Singh A, Marcilla I, Luquin MR, Papa SM (2014) Modeling Parkinson's disease in monkeys for transla- tional studies, a critical analysis. Exp Neurol 256:133-143.

27. Weatherall D (2006) The use of non-human primates in research. Academy of Medical Sciences, London.

28. Jeong HS, Lee SR, Kim JE, Lyoo IK, Yoon S, Namgung E, Chang KT, Kim BS, Yang S, Im JJ, Jeon S, Kang I, Ma J, Chung YA, Lim SM (2018) Brain structural changes in cynomolgus monkeys administered with 1-methyl-4-phenyl-1,2,3,6tetrahydropyridine: a longitudinal voxel-based morphometry and diffusion tensor imaging study. PLoS One 13:e0189804.

29. Won J, Yi KS, Choi CH, Jeon CY, Seo J, Kim K, Yeo HG, Park J, Kim YG, Jin YB, Koo BS, Lim KS, Lee S, Kim KJ, Choi WS, Park SH, Kim YH, Huh JW, Lee SR, Cha SH, Lee Y (2020) Assessment of hand motor function in a non-human primate model of ischemic stroke. Exp Neurobiol 29:300-313.

30. Imbert C, Bezard E, Guitraud S, Boraud T, Gross CE (2000) Comparison of eight clinical rating scales used for the assessment of MPTP-induced Parkinsonism in the Macaque monkey. J Neurosci Methods 96:71-76.

31. Kurlan R, Kim MH, Gash DM (1991) Oral levodopa dose-response study in MPTP-induced HemiParkinsonian monkeys: assessment with a new rating scale for monkey Parkinsonism. Mov Disord 6:111-118.

32. Kazumata K, Dhawan V, Chaly T, Antonini A, Margouleff C, Belakhlef A, Neumeyer J, Eidelberg D (1998) Dopamine transporter imaging with fluorine-18-FPCIT and PET. J Nucl Med 39:1521-1530.

33. Puschmann A, Wszolek ZK (2011) Diagnosis and treatment of common forms of tremor. Semin Neurol 31:65-77.

34. Bhidayasiri R (2005) Differential diagnosis of common tremor syndromes. Postgrad Med J 81:756-762.

35. Deuschl G, Bain P, Brin M (1998) Consensus statement of the movement disorder society on tremor. Ad Hoc Scientific Committee. Mov Disord 13 Suppl 3:2-23.

36. Dirkx MF, Zach H, Bloem BR, Hallett M, Helmich RC (2018) The nature of postural tremor in Parkinson disease. Neurology 90:e1095-e1103.

37. Emborg ME, Tetrud JW, Moirano J, McLaughlin WW, Bankiewicz KS (2003) Rest tremor in rhesus monkeys with MPTP-induced parkinsonism. Front Biosci 8:a148-a154.

38. Pan MK, Ni CL, Wu YC, Li YS, Kuo SH (2018) Animal models of tremor: relevance to human tremor disorders. Tremor Other Hyperkinet Mov (NY) 8:587.

39. Bergman H, Raz A, Feingold A, Nini A, Nelken I, Hansel D, Ben-Pazi H, Reches A (1998) Physiology of MPTP tremor. Mov Disord 13 Suppl 3:29-34.

40. Oiwa Y, Eberling JL, Nagy D, Pivirotto P, Emborg ME, Bankiewicz KS (2003) Overlesioned HemiParkinsonian non human 
primate model: correlation between clinical, neurochemical and histochemical changes. Front Biosci 8:a155-a166.

41. German DC, Dubach M, Askari S, Speciale SG, Bowden DM (1988) 1-Methyl-4-phenyl-1,2,3,6-tetrahydropyridine-induced Parkinsonian syndrome in Macaca fascicularis: which midbrain dopaminergic neurons are lost? Neuroscience 24:161-174.

42. Deutch AY, Elsworth JD, Goldstein M, Fuxe K, Redmond DE Jr, Sladek JR Jr, Roth RH (1986) Preferential vulnerability of A8 dopamine neurons in the primate to the neurotoxin 1-methyl-4-phenyl-1,2,3,6-tetrahydropyridine. Neurosci Lett 68:51-56.

43. Choudhury GR, Daadi MM (2018) Charting the onset of Parkinson-like motor and non-motor symptoms in nonhuman primate model of Parkinson's disease. PLoS One 13:e0202770.

44. Taylor JR, Elsworth JD, Roth RH, Sladek JR Jr, Redmond DE $\operatorname{Jr}$ (1990) Cognitive and motor deficits in the acquisition of an object retrieval/detour task in MPTP-treated monkeys. Brain 113 (Pt 3):617-637.

45. McEntire CR, Choudhury GR, Torres A, Steinberg GK, Red- mond DE Jr, Daadi MM (2016) Impaired arm function and finger dexterity in a nonhuman primate model of stroke: motor and cognitive assessments. Stroke 47:1109-1116.

46. Tetrud JW, Langston JW (1992) Tremor in MPTP-induced Parkinsonism. Neurology 42:407-410.

47. Dirkx MF, den Ouden HE, Aarts E, Timmer MH, Bloem BR, Toni I, Helmich RC (2017) Dopamine controls Parkinson's tremor by inhibiting the cerebellar thalamus. Brain 140:721734.

48. Caligiore D, Helmich RC, Hallett M, Moustafa AA, Timmermann L, Toni I, Baldassarre G (2016) Parkinson's disease as a system-level disorder. NPJ Parkinsons Dis 2:16025.

49. Lang EJ, Sugihara I, Llinás R (1996) GABAergic modulation of complex spike activity by the cerebellar nucleoolivary pathway in rat. J Neurophysiol 76:255-275.

50. Galvan A, Wichmann T (2007) GABAergic circuits in the basal ganglia and movement disorders. Prog Brain Res 160:287-312.

51. Pagano G, Politis M (2018) Molecular imaging of the serotonergic system in Parkinson's disease. Int Rev Neurobiol 141:173-210. 